The Astrophysical Journal, 251:L27-L31, 1981 December 1

(1) 1981. The American Astronomical Society. All rights reserved. Printed in U.S.A.

\title{
THE ISOTOPIC COMPOSITION OF COSMIC RAY B, C, N, AND O NUCLEI
}

\author{
R. A. Mewaldt, J. D. Spalding, E. C. Stone, and R. E. Vogt \\ California Institute of Technology \\ Received 1981 June 29; accepted 1981 August 13
}

\begin{abstract}
We report new high resolution measurements of the elemental and isotopic composition of galactic cosmic ray $\mathrm{B}, \mathrm{C}, \mathrm{N}$, and $\mathrm{O}$ nuclei with $\sim 30$ to $\sim 130 \mathrm{MeV}^{\text {nucleon }}{ }^{-1}$. These observations place limits on the isotopic composition of the cosmic ray source and restrict possible models of cosmic ray origin and propagation. In particular, we find that $\mathrm{N}$ is significantly depleted in the cosmic ray source with respect to the solar system and local interstellar medium, a result inconsistent with models in which a majority of cosmic rays are accelerated interstellar medium material.
\end{abstract}

Subject headings: cosmic rays: abundances - cosmic rays: general

\section{INTRODUCTION}

Recent high resolution measurements have shown that the neutron-rich isotopes of $\mathrm{Ne}, \mathrm{Mg}$, and $\mathrm{Si}$ are enhanced in the cosmic ray source by factors of $\sim 1.5$ to $\sim 4$ relative to their solar system abundances (see, e.g., Mewaldt et al. 1980; Wiedenbeck and Greiner 1981a, $1981 b)$, providing evidence that the nucleosynthesis of cosmic rays has differed from that of the bulk of solar system matter. There is evidence from millimeter-wave observations (Penzias 1980; Wannier 1980) that the abundances of interstellar $\mathrm{C}, \mathrm{N}$, and $\mathrm{O}$ isotopes also differ from those in the solar system, indicating the importance of studying these isotopes at the cosmic ray source. This study is difficult, however, because the observed cosmic ray abundances of the neutron-rich $\mathrm{C}$, $\mathrm{N}$, and $\mathrm{O}$ isotopes are expected to be dominated by spallation products produced during passage through the interstellar medium (ISM). But for the-same reason, these isotopes also provide tests of cosmic ray propagation models.

An important question to be addressed by isotope studies is whether cosmic rays represent recent supernova ejecta, accelerated either during the explosion or from the remnant, or whether they represent ISM material, possibly accelerated by interstellar shock waves. The abundance of nitrogen at the source has been suggested as a test of these alternatives (Silberberg, Shapiro, and Tsao 1975). This abundance is best determined by nitrogen isotope studies.

In this Letter we report new high resolution measurements of cosmic ray $\mathrm{B}, \mathrm{C}, \mathrm{N}$, and $\mathrm{O}$ in which the isotopes are separately resolved and the resulting abundances limited by statistical rather than systematic uncertainties.

\section{OBSERVATIONS}

These observations were made with the Caltech Heavy Isotope Spectrometer Telescope (HIST) on ISEE 3 during quiet-time periods between 1978 August 13 and 1978 December 1 . We have limited the energy interval for analysis to $\geq 30 \mathrm{MeV}$ nucleon ${ }^{-1}$ in order to exclude the anomalous enhancements in the $\mathrm{N}$ and $\mathrm{O}$ spectra observed at lower energy (see, e.g., Gloeckler 1979). Details of the method of resolving isotopes in the HIST solid-state detector telescope are discussed in Mewaldt et al. (1980), and a preliminary version of this report is presented in Mewaldt et al. (1981a, 1981b).

In the observed mass distributions shown in Figure 1, the measured mass resolution ranges from $0.07 \mathrm{amu}$ at $\mathrm{B}$ to $0.15 \mathrm{amu}$ at $\mathrm{O}$, allowing mass assignments to be made on an individual particle basis. The data in Figure 1 represent the best mass resolution so far achieved for heavy cosmic ray isotopes. Unfortunately, a component failure in the HIST readout logic on 1978 December 1 (which reduced the number of data bits transmitted to Earth) limited the period during which high resolution data such as these could be accumulated.

Table 1 summarizes the element and isotope ratios that we obtain. Our element abundances are in good agreement with other satellite observations at similar energies (Garcia-Munoz and Simpson 1979). Figure 2 compares our measured isotope abundances with other selected observations and with the results of cosmic ray propagation and solar modulation calculations (kindly provided by M. E. Wiedenbeck). The calculations assume a standard leaky-box propagation model with a mean path length $\lambda=5.5 \mathrm{~g} \mathrm{~cm}^{-2}$ of interstellar matter (with $\mathrm{He} / \mathrm{H}=0.1$ ) and solar modulation with a mean energy loss $\Phi=300 \mathrm{MeV}^{\text {nucleon }}{ }^{-1}$. 


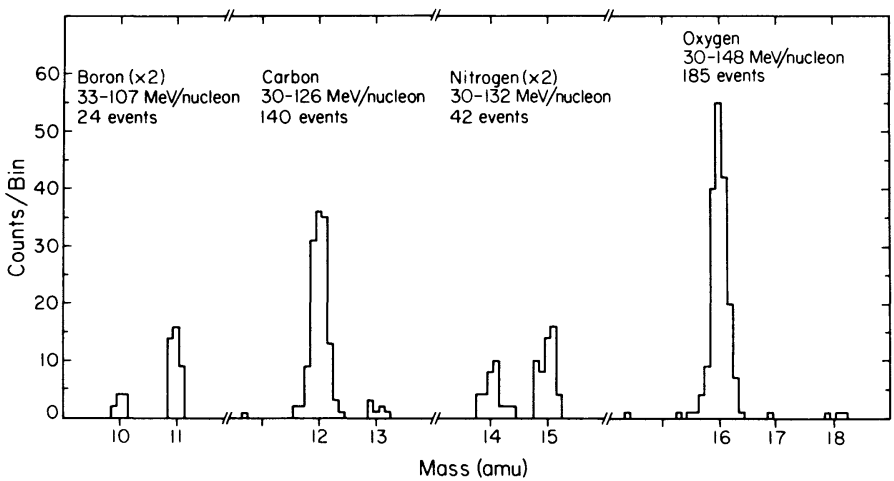

Fig. 1.-Mass histograms of $\mathrm{B}, \mathrm{C}, \mathrm{N}$, and $\mathrm{O}$ nuclei in the indicated energy intervals. Note that the boron and nitrogen distributions have been scaled up by a factor of 2 . Event totals at rare isotopes are: ${ }^{10} \mathrm{~B}=5,{ }^{13} \mathrm{C}=7,{ }^{17} \mathrm{O}=1$, and ${ }^{18} \mathrm{O}=3$.

TABLE 1

OBSERVED ELEMENT AND ISOTOPE RATIOS

\begin{tabular}{|c|c|c|c|c|}
\hline Element & $\begin{array}{c}\text { Energy } \\
\text { Interval } \\
\left(\mathrm{MeV} \text { nucleon }^{-1}\right)\end{array}$ & $\begin{array}{c}\text { Observed } \\
\text { Abundance }^{\mathrm{a}}\end{array}$ & $\begin{array}{l}\text { Isotope } \\
\text { Ratio }\end{array}$ & $\begin{array}{l}\text { Observed } \\
\text { Ratio }^{\mathbf{a}}\end{array}$ \\
\hline B......... & $33-107$ & $0.22\left\{\begin{array}{l}+0.06 \\
-0.05\end{array}\right.$ & ${ }^{10} \mathrm{~B} / \mathrm{B}$ & $0.19\left\{\begin{array}{l}+0.11 \\
-0.05\end{array}\right.$ \\
\hline $\mathrm{C} \ldots \ldots \ldots$ & $30-126$ & $0.99 \pm 0.12$ & ${ }^{13} \mathrm{C} / \mathrm{C}$ & $0.052\left\{\begin{array}{l}+0.029 \\
-0.011\end{array}\right.$ \\
\hline $\mathrm{N} .$. & $30-132$ & $0.26 \pm 0.05$ & ${ }^{15} \mathrm{~N} / \mathrm{N}$ & $0.63 \pm 0.07$ \\
\hline \multirow[t]{2}{*}{ O.......... } & $30-148$ & $\equiv 1.00$ & ${ }^{17} \mathrm{O} /{ }^{16} \mathrm{O}$ & $0.006\left\{\begin{array}{l}+0.013 \\
-0.006\end{array}\right.$ \\
\hline & & & ${ }^{18} \mathrm{O} /{ }^{16} \mathrm{O}$ & $0.018\left\{\begin{array}{l}+0.017 \\
-0.006\end{array}\right.$ \\
\hline
\end{tabular}

${ }^{\mathrm{a}} 68 \%$ confidence intervals.

\section{INTERPRETATION OF THE OBSERVATIONS}

Our observations of ${ }^{13} \mathrm{C} / \mathrm{C},{ }^{17} \mathrm{O} /{ }^{16} \mathrm{O}$, and ${ }^{18} \mathrm{O} /{ }^{16} \mathrm{O}$ are consistent with the calculated ratios and with most of the other results shown in Figure 2, while our ${ }^{10} \mathrm{~B} / \mathrm{B}$ measurement is marginally below the calculated value. Table 2 includes limits on the source abundances of the $\mathrm{C}, \mathrm{N}$, and $\mathrm{O}$ isotopes, assuming $\lambda=5.5 \mathrm{~g} \mathrm{~cm}^{-2}$ and $\Phi=300 \mathrm{MeV}$ nucleon $^{-1}$. Larger values for either $\lambda$ or $\Phi$ (see below) would lead to smaller limits on these ratios. While we find no evidence for nonsolar source abundances of the neutron-rich isotopes of $\mathrm{C}$ and $\mathrm{O}$, neither this or the other recent satellite study included in Table 2 rules out the possibility of enhancements of a factor of 2 (or, in some cases, even more) in their source abundances. Note that although the cosmic ray results for the $\mathrm{C}$ and $\mathrm{O}$ isotopes are not yet sufficiently precise to discriminate between the solar system and ISM, only modest improvements in statistical accuracy and knowledge of the relevant cross sections are necessary to do so (Wiedenbeck and Greiner 1981 $a, 1981 b$ ).

Figure 2 also presents the $\mathrm{N}$ observations, among which there are considerable disagreements, with re- ported ${ }^{15} \mathrm{~N} / \mathrm{N}$ ratios ranging from $\sim 0.3$ to $\sim 0.6$. Some of these disagreements are undoubtedly due in part to marginal isotope resolution in some of the earlier experiments. Our measured value $\left({ }^{15} \mathrm{~N} / \mathrm{N}=0.63 \pm 0.07\right)$ is $>0.5$ at the $95 \%$ confidence level, and significantly $(\sim 3 \sigma)$ greater than the calculated ratio in Figure 2, as are several of the other measurements (Preszler et al. 1975; Hagen, Fisher, and Ormes 1977; Webber, Kish, and Simpson 1979; Wiedenbeck et al. 1979). The difference between the calculated and observed values implies that either ${ }^{15} \mathrm{~N}$ at the source is significantly enhanced over its solar system abundance $\left({ }^{15} \mathrm{~N} / \mathrm{N}=\right.$ 0.0037; Cameron 1980), or the cosmic ray propagation calculation is in need of revision. In the following discussion we assume that the source abundance of ${ }^{15} \mathrm{~N}$ is negligible. The extent to which the observed ${ }^{15} \mathrm{~N} / \mathrm{N}$ ratio deviates from that expected from fragmentation alone is then a measure of the ${ }^{14} \mathrm{~N}$ abundance at the source.

In a recent study of the CNO isotopes, Guzik (1981) pointed out that the ${ }^{14} \mathrm{~N}$ and ${ }^{15} \mathrm{~N}$ production cross sections from ${ }^{16} \mathrm{O}$ have not been directly measured below $\sim 2 \mathrm{GeV}$ nucleon $^{-1}$. He suggested revised cross 


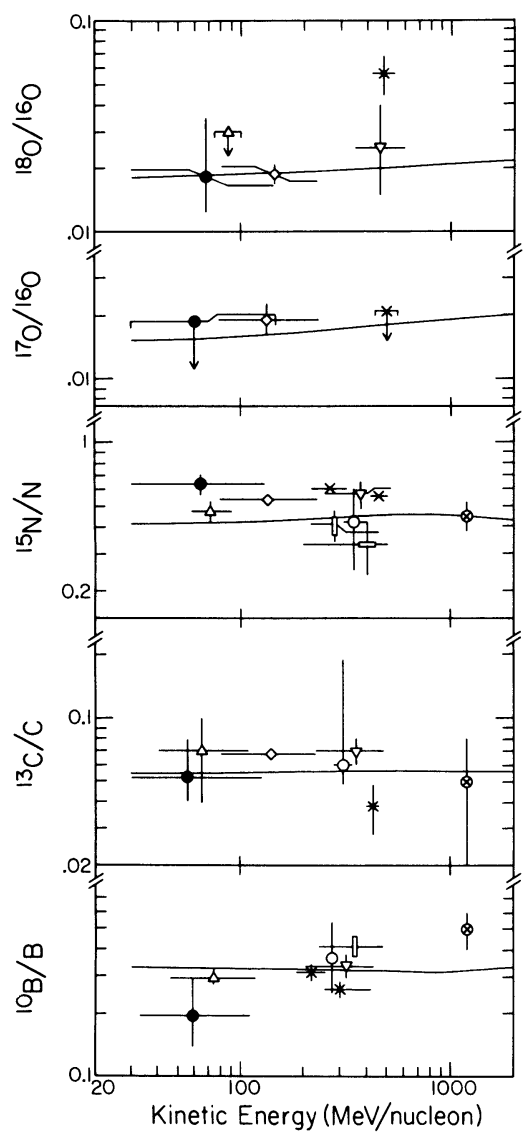

Fig. 2.-A comparison of measured and calculated isotopic ratios. Data points: filled circle, this work; triangle, Guzik (1981), Garcia-Munoz, Mason, and Simpson (1977); diamond, Weidenbeck et al. (1979), Wiedenbeck and Greiner (1981 $a, 1981 b)$; vertical rectangle, Buffington, Orth, and Mast (1978); open circle, Zumberge (1981); inverted triangle, Hagen, Fisher, and Ormes (1977), Fisher et al. (1976); cross, Preszler et al. (1975), Webber and Kish (1979), Webber, Kish, and Simpson (1979); horizontal rectangle, Bjarle et al. (1977); circled cross, Dwyer (1978). The curves were calculated using source elemental abundances from Silberberg, Tsao, and Shapiro (1976), isotopic abundances from Cameron (1980), and cross sections from Tsao and Silberberg (1979).

sections based on scaling $p+{ }^{12} \mathrm{C} \rightarrow{ }^{10,11} \mathrm{~B}$ measurements. Thus the predicted ${ }^{15} \mathrm{~N} / \mathrm{N}$ ratio for fragmentation alone is 0.58 or 0.65 , depending on whether Tsao and Silberberg (1979) or Guzik cross sections are used. Since both of the predictions are consistent with our observed ratio $(0.63 \pm 0.07)$, it is possible that all of the observed nitrogen results from fragmentation and none is from the source.

Of course, the addition of a finite source abundance of ${ }^{14} \mathrm{~N}$ yields smaller predicted ${ }^{15} \mathrm{~N} / \mathrm{N}$ ratios. However, our observed $84 \%$ confidence limit of ${ }^{15} \mathrm{~N} / \mathrm{N} \geq 0.56$ restricts such source abundances to ${ }^{14} \mathrm{~N} / \mathrm{O} \leq 0.01$ for the Tsao and Silberberg cross sections and ${ }^{14} \mathrm{~N} / \mathrm{O} \leq$ 0.03 for Guzik's cross sections (see Figs. 9 and 10 of
Guzik 1981), using the standard propagation model. These limits are rather insensitive to the details of the model, so that for a broad range of assumed path lengths $\left(4 \leq \lambda \leq 8 \mathrm{~g} \mathrm{~cm}^{-2}\right)$ and solar modulation (100 $\leq \Phi \leq 400 \mathrm{MeV}$ nucleon ${ }^{-1}$ ) we find ${ }^{14} \mathrm{~N} / \mathrm{O} \leq 0.04$. There is less than a $2 \%$ probability that the source ${ }^{14} \mathrm{~N} / \mathrm{O}$ ratio could be as large as 0.10 for the above ranges of $\lambda$ and $\Phi$. Our limit on the ${ }^{14} \mathrm{~N}$ source abundance is consistent with that of Webber, Kish, and Simpson (1979) who found $\mathrm{N} / \mathrm{O}=0.028 \pm 0.009$, but is at the lower limit of Guzik's value of $0.07 \pm 0.03$. Note that our observed ${ }^{14} \mathrm{~N} / \mathrm{O}$ and ${ }^{15} \mathrm{~N} / \mathrm{O}$ ratios are $0.10 \pm 0.03$ and $0.17 \pm 0.04$, respectively. Thus a source ratio of ${ }^{14} \mathrm{~N} / \mathrm{O} \geq 0.10$, typical of the solar system and ISM, would require that the secondary contribution to ${ }^{14} \mathrm{~N} / \mathrm{O}$ be $\leq 0.03$, or $\lesssim 20 \%$ of that to ${ }^{15} \mathrm{~N} / \mathrm{O}$. Since the calculated ${ }^{14} \mathrm{~N}$ production is $54-72 \%$ of that for ${ }^{15} \mathrm{~N}$, the relative cross sections would have to be in error by a factor of $\gtrsim 2.7$.

The observed N/O element ratio provides an additional constraint on the propagation model (Guzik 1981). For example, the observed element ratio $\mathrm{N} / \mathrm{O}=0.25 \pm$ 0.01 (Garcia-Munoz and Simpson 1979) combined with our ${ }^{15} \mathrm{~N} / \mathrm{N}$ isotope ratio requires $\lambda \geq 6 \mathrm{~g} \mathrm{~cm}^{-2}$ for $\Phi \leq$ $400 \mathrm{MeV}$ nucleon ${ }^{-1}$ using Guzik's cross sections. In addition, the $\mathrm{N} / \mathrm{O}$ and ${ }^{15} \mathrm{~N} / \mathrm{N}$ observations allow a limit of $\leq 0.09$ to be placed on the ${ }^{15} \mathrm{~N} / \mathrm{O}$ ratio at the source, although there are presently no limits that we can place on the source ${ }^{15} \mathrm{~N} /{ }^{14} \mathrm{~N}$ ratio.

\section{DISCUSSION}

Although there is as yet no strong evidence for nonsolar abundances of the neutron-rich isotopes of $\mathrm{C}, \mathrm{N}$, and $\mathrm{O}$ in cosmic rays, the present observations do restrict possible explanations for the observed enhancements in the neutron-rich isotopes of $\mathrm{Ne}, \mathrm{Mg}$, and $\mathrm{Si}$. Wiedenbeck and Greiner (1981a) concluded that their ${ }^{18} \mathrm{O} /{ }^{16} \mathrm{O}$ observation was inconsistent with one of the Woosley and Weaver (1981) models in which stars with $M \sim 10 M_{\odot}$ eject a shell rich in ${ }^{22} \mathrm{Ne}$ and ${ }^{18} \mathrm{O}$. Another suggested model, which invokes explosive processing of the external hydrogen-rich envelope of novae or supernovae (Cassé, Meyer, and Reeves 1979), also predicts a ${ }^{13} \mathrm{C}$ enhancement which appears to be several times larger than allowed by the present observations.

Silberberg, Shapiro, and Tsao (1975) have previously pointed out that the $\mathrm{N}$ abundance can serve as a test of cosmic ray origin models, with a low $\mathrm{N}$ abundance favoring models in which the cosmic ray source abundances are similar to those of supernova ejecta, rather than to those of the ISM. Hainebach, Norman, and Schramm (1976) have discussed a model in which a mixture of supernova and ISM material leads to a low N/O ratio. Note that in solar system (Cameron 1980) and ISM material (Wannier 1980) the $\mathrm{N} / \mathrm{O}$ ratio is essentially ${ }^{14} \mathrm{~N} /{ }^{16} \mathrm{O}$. 
TABLE 2

ISOTOPE RATIOS

\begin{tabular}{|c|c|c|c|c|c|c|}
\hline \multirow[b]{2}{*}{$\begin{array}{l}\text { ISOTOPE } \\
\text { RATIO }\end{array}$} & \multicolumn{3}{|c|}{ Cosmic Ray Source } & \multicolumn{2}{|c|}{ INTERSTELLAR MEDIUM $^{a}$} & \multirow[b]{2}{*}{$\begin{array}{c}\text { SOLAR } \\
\text { SYSTEM }^{b}\end{array}$} \\
\hline & $\begin{array}{l}\text { This } \\
\text { Work }\end{array}$ & $\begin{array}{l}\text { Guzik } \\
(1981)\end{array}$ & $\begin{array}{c}\text { Wiedenbeck } \\
\text { and Greiner } \\
(1981 a ; 1981 b)\end{array}$ & $\begin{array}{c}\text { Galactic } \\
\text { Ring }\end{array}$ & $\begin{array}{c}\text { Galactic } \\
\text { Center }\end{array}$ & \\
\hline${ }^{13} \mathrm{C} / \mathrm{C} \ldots \ldots$ & $\leq 0.044^{\mathrm{c}}$ & $\ldots$ & $0.021 \pm 0.006^{c}$ & 0.017 & 0.038 & 0.0111 \\
\hline${ }^{17} \mathrm{O} /{ }^{16} \mathrm{O} \ldots$ & $\leq 0.012^{\mathrm{c}}$ & $\ldots$ & $0.004\left\{\begin{array}{c}+0.004^{\mathrm{c}} \\
-0.003\end{array}\right.$ & 0.0006 & 0.0010 & 0.00037 \\
\hline $\begin{array}{l}{ }^{18} \mathrm{O} /{ }^{16} \mathrm{O} \ldots \\
{ }^{14} \mathrm{~N} /{ }^{16} \mathrm{O} \ldots \\
{ }^{15} \mathrm{~N} /{ }^{16} \mathrm{O} \ldots\end{array}$ & $\begin{array}{l}\leq 0.020^{c} \\
\leq 0.04 \\
\leq 0.09\end{array}$ & $\begin{array}{c}0.07 \pm 0.03 \\
\ldots\end{array}$ & $\begin{array}{c}0.002 \pm 0.002^{\mathrm{c}} \\
\ldots \\
\ldots\end{array}$ & $\begin{array}{c}\quad 0.0020 \\
\sim 0.10^{\mathrm{d}} \\
\quad \ldots\end{array}$ & $\begin{array}{c}0.0033 \\
\ldots \\
\ldots\end{array}$ & $\begin{array}{l}0.00204 \\
0.1255 \\
0.00046\end{array}$ \\
\hline
\end{tabular}

\footnotetext{
${ }^{\text {a}}$ Wannier 1980. The galactic ring measurements are for galactic radii of $\sim 5$ to $\sim 13 \mathrm{kpc}$.

${ }^{\mathrm{b}}$ Cameron 1980.

${ }^{c}$ Includes only observational uncertainty. Wiedenbeck and Greiner estimate propagation uncertainties (dominated by cross section uncertainties) to be 0.002 to 0.005 for ${ }^{17.18} \mathrm{O} /{ }^{16} \mathrm{O}$ and 0.012 to 0.017 for ${ }^{13} \mathrm{C} / \mathrm{C}$.

${ }^{\mathrm{d}}$ See text.
}

The ${ }^{14} \mathrm{~N}$ source abundance that we determine $\left({ }^{14} \mathrm{~N} /{ }^{16} \mathrm{O} \leq 0.04\right)$ is significantly less than in the solar system (see Table 2). Ross and Aller (1976) give a solar abundance ratio of $\mathrm{N} / \mathrm{O}=0.13 \pm 0.05$ based on a variety of observations. Recent solar spectroscopic observations include $\mathrm{N} / \mathrm{O}=0.12 \pm 0.04$ for the photosphere (Lambert 1978) and $\mathrm{N} / \mathrm{O}=0.14 \pm 0.01$ for the corona (McKenzie et al. 1978). For solar energetic particles, Cook, Stone, and Vogt (1980) find N/O $=0.12$ \pm 0.01 . Thus observations of this ratio in the solar system appear to be reasonably self-consistent.

The solar system abundances are thought to be representative of the local ISM $\sim 5$ billion years ago. Direct observations of $\mathrm{N} / \mathrm{O}$ in the ISM include the work of Hawley (1978) who surveyed $13 \mathrm{H}$ II regions with galactic radii ranging from $\sim 8$ to $\sim 14 \mathrm{kpc}$. He found $\mathrm{N} / \mathrm{O}$ ratios ranging from $\sim 0.05$ to $\sim 0.20$ with a negative radial gradient in the Galaxy of $d(\log \mathrm{N} / \mathrm{O}) / d r=$ $-0.06 \pm 0.02 \mathrm{kpc}^{-1}$. At the Sun's location of $\sim 10 \mathrm{kpc}$ the typical $\mathrm{N} / \mathrm{O}$ ratio is $\sim 0.10$, in agreement with ob- servations of the Orion Nebula at $10.4 \mathrm{kpc}$ (Peimbert and Torres-Peimbert 1977). Thus it appears that nitrogen is depleted in the cosmic ray source relative to the local ISM by at least a factor of 2 . It is, of course, possible that some mechanism of preferential acceleration has depleted $\mathrm{N}$ relative to $\mathrm{O}$ in cosmic rays. However, recent models that invoke an acceleration efficiency that depends on the first ionization potential or related atomic parameters to explain the elemental composition of cosmic rays do not predict a significant effect on the N/O ratio (cf. Cassé and Goret 1978). Thus we conclude that our ${ }^{14} \mathrm{~N} /{ }^{16} \mathrm{O}$ limit is inconsistent with models in which a majority of cosmic rays are accelerated ISM material.

We thank M. E. Wiedenbeck for the use of his cosmic ray propagation calculations and for discussions of their interpretation. This work was supported in part by NASA under contract NAS 5-20721 and grant NGR 05-002-160.

\section{REFERENCES}

Bjarle, C., Herrström, N.-Y., Jacobsson, L., Jönsson, G., and Kristiansson, K. 1977, Proc. 15th Internat. Cosmic Ray Conf. (Plovdiv), 1, 313.

Buffington, A., Orth, C. D., and Mast, T. S. 1978, Ap. J., 226, 355.

Cameron, A. G. W. 1980, Harvard-Smithsonian Center for Astrophysics, preprint No. 1357.

Cassé, M., and Goret, P. 1978, Ap. J., 221, 703.

Cassé, M., Meyer, J. P., and Reeves, H. 1979, Proc. 16th Internat. Cosmic Ray Conf. (Kyoto), 12, 114.

Cook, W. R., Stone, E. C., and Vogt, R. E. 1980, Ap. J. (Letters), 238, L97.

Dwyer, R. 1978, Ap. J., 224, 691.

Fisher, A. J., Hagen, F. A., Maehl, R. C., Ormes, J. F., and Arens, J. F. 1976, Ap. J., 205, 938

Garcia-Munoz, M., Mason, G. M., and Simpson, J. A. 1977, Proc. 15th Internat. Cosmic Ray Conf. (Plovdiv), 1, 301.

Garcia-Munoz, M., and Simpson, J. A. 1979, Proc. 16th Internat. Cosmic Ray Conf. (Kyoto), 1, 270.

Gloeckler, G. 1979, Rev. Geophys. Space Phys., 17, 569.

Guzik, T. G. 1981, Ap. J., 244, 695.

Hagen, F. A., Fisher, A. J., and Ormes, J. F. 1977, Ap. J., 212, 262.
Hainebach, K. L., Norman, E. B., and Schramm, D. N. 1976, Ap. $J ., 203,245$.

Hawley, S. A. 1978, Ap. J., 224, 417.

Lambert, D. L. 1978, M.N.R.A.S., 182, 249.

McKenzie, D. L., Rugge, H. R., Underwood, J. H., and Young, R. M. 1978, $A p . J ., 221,342$.

Mewaldt, R. A., Spalding, J. D., Stone, E. C., and Vogt, R. E. 1980, Ap. J. (Letters), 235, L95. 68. (Paris).

Peimbert, M., and Torres-Peimbert, S. 1977, M.N.R.A.S., 179, 217

Penzias, A. A. 1980, Science, 208, 663.

Preszler, A. M., Kish, J. C., Lezniak, J. A., Simpson, G., and Webber, W. R. 1975, Proc. 14th Internat. Cosmic Ray Conf. (Munich), 12, 4096.

Ross, J. E., and Aller, L. H. 1976, Science, 191, 1223.

Silberberg, R., Shapiro, M. M., and Tsao, C. H. 1975, Proc. 14th Internat. Cosmic Ray Conf. (Munich), 2, 451. 
Silberberg, R., Tsao, C. H., and Shapiro, M. M. 1976, in Spallation Nuclear Reactions and their Applications, ed. B. S. P. Shen and M. Merker (Dordrecht: Reidel), p. 49.

Tsao, C. H., and Silberberg, R. 1979, Proc. 16th Internat. Cosmic Ray Conf. (Kyoto), 2, 202

Wannier, P. G. 1980, Ann. Rev. Astr. Ap., 18, 399.

Webber, W. R., and Kish, J. 1979, Proc. 16th Internat. Cosmic Ray Conf. (Kyoto), 1, 389.

Webber, W. R., Kish, J., and Simpson, G. 1979, Proc. 16th Internat. Cosmic Ray Conf. (Kyoto), 1, 424.
Wiedenbeck, M. E., and Greiner, D. E. 1981a, Phys. Rev. Letters, 46, 682 .

1981 b, Ap. J. (Letters), 247, L119.

Wiedenbeck, M. E., Greiner, D. E., Bieser, F. S., Crawford, H. J., Heckman, H., and Lindstrom, P. J. 1979, Proc. 16th Internat. Cosmic Ray Conf. (Kyoto), 1, 412 .

Woosley, S. E., and Weaver, T. A. 1981, Ap. J., 243, 651

Zumberge, J. F. 1981, Ph.D. thesis, California Institute of Technology.

Richard A. Mewaldt, John D. Spalding, Edward C. Stone, and Rochus E. Vogt: California Institute of Technology, Mail Code 220-47, Pasadena, CA 91125 